third day of life since fetal excretory functions are performed mainly by the placenta with minimal contribution by the fetal kidney. After placental separation, a rapid increase in the renal circulation occurs over the first days of life (6). We have found that after an initial extrauterine adaptation of glomerular function in the first 2-3 days of life, no further functional acceleration occurs in the immediate postnatal period despite continued challenges imposed on the kidney during postnatal life.

Our findings of remarkably low $C_{i n}$ for the infants of 25-30 weeks' gestation point out the necessity for modifying fluid and drug administration to small premature infants, but add the encouraging note that even $545-\mathrm{g}$ infants have a predictable, sufficient glomerular filtration rate $\left(C_{i n}\right)$ for survival. It is not known whether the rate of maturation in the first few hours of life is as rapid for the markedly premature infant as that demonstrated in infants of higher gestational age. This aspect of ontogeny in renal function requires further investigation.

\section{SUMMARY}

The inulin clearances of 38 healthy infants varying in gestational age from 25 to 42 weeks were studied by the constant infusion method. The validity of the constant infusion for $C_{i n}$ was confirmed. Inulin clearance was directly proportional to gestational age when measured at 2-3 days of age. Inulin clearance was proportional to conceptual age (gestational age and postnatal age) when measured at 1-9 weeks of age. There was no functional acceleration of inulin clearance when infants of a particular gestational age studied at 2-3 days of life were compared with those attaining a similar conceptual age (gestational plus postnatal age).

Copyright ( 1976 International Pediatric Research Foundation, Inc.

\section{REFERENCES AND NOTES}

1. Barnett, H. L., Hare, W. K., McNamara, H., and Hare, R. S.: Influence of postnatal age on kidney function of premature infants. Proc. Soc. Exp. Biol. A. Med., 69: 55 (1948).

2. Cole, B. R., Giangiacomo, J., Ingelfinger, J., and Robson, A.: Measurement of renal function without urine collection. N. Engl. J. Med., 287: 1109 (1972).

3. Dubowitz, L. M., Dubowitz, V., and Goldberg, C.: Clinical assessment of gestational age in newborn infants. J. Pediat., 77 : 1 (1970).

4. Edelmann, Jr., C. M.: Pediatric nephrology. Pediatrics, 51: 854 (1973).

5. Guignard, J. P., Torrado, A., Da Cunha, O., and Gautier, E.: Glomerular filtration rate in the first three weeks of life. J. Pediat., 87: 268 (1975).

6. Oh, W., Oh, M. A., and Lind, J.: Renal function and blood volume in newborn infants related to placental transfusion. Acta Paediat. Scand., 56: 197 (1966).

7. Olbing, H., Blaufox, M. D., Aschinberg, L. C., Silkalns, G. I., Bernstein, J., Spitzer, A., and Edelmann, C. M., Jr.: Postnatal changes in renal glomerular blood flow distribution in puppies. J. Clin. Invest., 53: 2885 (1973).

8. Robillard, J. E., Kulvinskas, C., Sessions, C., Burmeister, L., and Smith, F. G.: Maturational changes in the fetal glomerular filtration rate. Amer. J. Obstet. Gynecol., 122: 601 (1975).

9. Roe, J. H., Epstein, J. H., and Goldstein, N. P.: A photometric method for the determination of inulin in plasma and urine. J. Biol. Chem., 178: 839 (1949).

10. Spitzer, A., and Brandis, M.: Functional and morphologic maturation of the superficial nephrons. J. Clin. Invest., 53: 279 (1974).

11. Vesterdal, J., and Tudvad, F.: Studies on the kidney function in premature and full term infants by estimation of the inulin and Para-aminohippurate clearance. Acta Paediat. Scand., 37: 429 (1949).

12. Purified Inulin Solution, Stone Arnar Company, Mt. Prospect, III.

13. Harvard Pump, Harvard Instrument Co.

14. The authors wish to thank Ruey-Yueh Ho for technical assistance in this study.

15. This paper was presented in part at the Society for Pediatric Research, Washington, D.C., May 3, 1974.

16. This research was supported in part by the General Research Support Grant P1549, National Institute of Health, Bethesda, Maryland.

17. The present address of Dr. W. Oh is: Women and Infants Hospital of Rhode Island, 50 Maude St., Providence, R.I. 02908 (USA).

18. Requests for reprints should be addressed to: R. D. Leake, M.D., Department of Pediatrics, Harbor General Hospital, 1000 W. Carson St., Torrance, Calif. 90509 (USA)

19. Accepted for publication February 17, 1976.

\title{
Child's Urinary Lithiasis Revealing a Complete Deficit in Adenine Phosphoribosyl Transferase
}

\author{
H. DEBRAY, ${ }^{11}$ P. CARTIER, A. TEMSTET, AND J. CENDRON \\ Hôpital Saint-Joseph, Service de Pediatrie, Paris, France
}

\section{Extract}

In one case of a urinary lithiasis, termed "uric lithiasis" on biochemical examination, the authors describe the symptomatology of a child with a complete deficit in adenine phosphoribosyl transferase. After more intensive investigation the calculi have been found to be composed of a new clinical compound: 2,8-hydroxyadenine.

\section{Speculation}

A diagnosis of infantile uric lithiasis should be accepted with certain reservations in the absence of a spectrophotometric examination of the calculus.
The pathogenesis of uric acid lithiasis, a rare disease in pediatrics, is uncertain. At Saint-Joseph's hospital in Paris, we observed a very young child with multiple recurring stones. After biochemical examination the stones were labeled as "uric lithiasis." This observation proved interesting for two reasons: it led to the discovery of a complete deficit of an enzyme associated with purine metabolism (APRTase). To our knowledge a complete deficit of APRTase has not been described. In addition, it led us to a more complete examination of the calculus by spectrophotometry. Our findings, to be described, indicate that a diagnosis of infantile uric lithiasis must be accepted with certain reservations in the absence of a spectrophotometric examination of the calculus. 


\section{CASE REPORT}

Pascal Tho. . . was 3.5 years old when he was first admitted to Saint-Joseph's hospital because he had formed renal stones.

\section{PAST HISTORY}

The child was born in France June 24, 1970. Childbirth was uneventful. There was no obstetrical or neonatal incident and the pregnancy was of normal duration. Weight at birth was $3.350 \mathrm{Kg}$ (skull circumference, $37 \mathrm{~cm}$; chest circumference, $34.5 \mathrm{~cm}$ ). The childhood course was unremarkable.

\section{FAMILY HISTORY (SCHEME I)}

The genealogy is as follows-sibling: Fabrice, 7 years old, stepbrother on the mother's side, healthy; mother; 27 years old, healthy; father: 28 years old, born in France, healthy, no previous history of gout, urinary lithiasis, or diabetes and no high blood pressure or obesity; a maternal uncle: possible gout; maternal grandfather: Italian extraction, 50 years old, hyperuricemic, is supposed to have recently passed a urinary calculus; maternal grandmother: born in France, is supposed to have passed one urinary calculus years ago, no past history of gout.

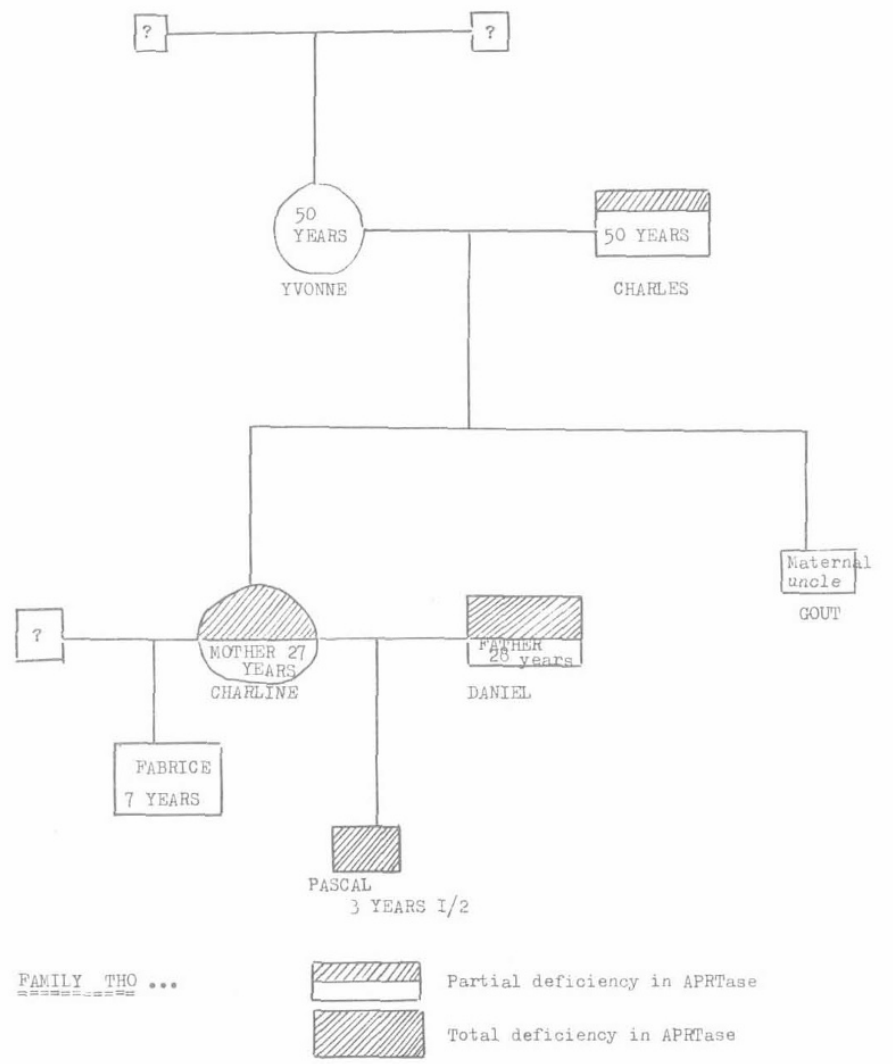

Scheme 1. Genealogical tree for the family Tho. . . .
HISTORY OF ILLNESS

In November 1972, Pascal, 2.5 years old, complained of dysuria. This was followed by the spontaneous passage of a calculus the size of a green pea. There was no hematuria.

He was admitted to a hospital in Paris where the calculus was believed to consist of uric acid; but further investigations were not made. A few days later, the child suffered from a urinary retention for a few hours which was followed by the emission of two more calculi. At the end of November 1972, several small stones were discovered by routine examination to be in the distal portion of the urethra. The child was admitted to Saint-Joseph's hospital for their surgical removal under general anesthesia. Intravenous urography did not show any other calculi: the right urinary tract appeared to be normal. However, there was a secretion delay and a moderate total dilation on the left. No gross congenital defect was observed. Cystography was normal. The urine was sterile. Laboratory data showed a normal serum phosphate and a slightly increased uric acid concentration (54 mg/liter).

No therapy was prescribed. The child was in good health until August 1973 when he developed a sudden rise of temperature. This was followed 15 days later by the emission of several fusiform calculi (more than 20). He experienced no dysuria nor hematuria.

In September 1973, Pascal developed painful dysuria. His mother felt a small hard nodule by palpating the penis. The child was readmitted again to the hospital.

Upon examination, Pascal, now 3.5 years old, was in good general condition, weighing $14.7 \mathrm{~kg}$ with a height of $96.5 \mathrm{~cm}$. The kidneys were not enlarged, nor was there any vesical distention. Miction was observed; the stream was weak but continuous with a terminal hematuria. On the following day Pascal spontaneously passed approximately 20 small calculi. X-ray of the kidneys did not reveal any opaque calculi and the right urinary tract appeared normal. Although the left kidney appeared to be smaller than the right, no enlargement was noticed. The bladder was regular. The urethra was slightly dilated and probably still contained a few calculi. The urine was sterile. Urinary $\mathrm{pH}$ varied between 6 and 9; however, a $\mathrm{pH}$ of 5 was recorded on three occasions. The 24-hr volume approximated $500 \mathrm{cc}$. The urinary electrolytes were normal. Blood urea nitrogen was $0.33 \mathrm{~g} /$ liter and endogenous serum creatinine was $5 \mathrm{mg} /$ liter. The corrected clearances were low: urea $26 \mathrm{ml} / \mathrm{min}$, creatinine $56 \mathrm{ml} / \mathrm{min}$. There was no microscopic hematuria. The calculi were analyzed and appeared to be simple uric acid stones. Uricemia was noted (64 and 44 $\mathrm{mg} /$ liter, a slightly high level for a child of this age). The uric acid concentration in the urine was $660 \mathrm{mg} /$ liter, equivalent to 150 $\mathrm{mg} / 24 \mathrm{hr}$. This is not an unexpected excretion. The corrected clearance of uric acid was $7.2 \mathrm{ml} / \mathrm{min}$, a normal rate. Column chromatography of the amino acids in urine was normal.

At this point, the consensus of opinion was that this case represented uric acid lithiasis in a young child the roles that the hyperuricemia in the setting of possible familial hyperuricemia played in Pascal's disease was uncertain. The recommended treatment was: vigorous ingestion of water 1.5 liters $/ 24 \mathrm{hr}$ except at night and alkalinization of the urine with sodium bicarbonate ( 2 $\mathrm{g} /$ day).

In February 1974, Pascal was reexamined in the clinic. He had no urinary trouble since he left, and no passage of calculi. The urinary $\mathrm{pH}$, checked by his mother, remained above 7. An

Table 1. Adenine phosphoribosyl transferase activity in red cells (nanomoles per $\mathrm{mg}$ hemoglobin per hr)

\begin{tabular}{|c|c|c|c|c|c|c|c|}
\hline & \multirow[b]{2}{*}{ Normal value } & \multicolumn{4}{|c|}{ Tho... } & \multicolumn{2}{|c|}{ Mal... } \\
\hline & & Pascal & Fabrice & Charline & Daniel & Yvonne & Charles \\
\hline Adenine & $26.9 \pm 4.9$ & 0.002 & 33.4 & 8.7 & 11.2 & 25.4 & 12.6 \\
\hline Hypoxanthine & $101 \pm 21$ & 126 & 128 & 104 & 97 & 100 & 122 \\
\hline Quanine & $164 \pm 40$ & 169 & 194 & 157 & 161 & 120 & 151 \\
\hline
\end{tabular}


evaluation of Pascal's family was performed in March 1974. We performed a quantitative analysis of serum uric acid, urine uric acid, serum and urine calcium, serum and urine phosphorus, blood urea nitrogen, serum creatinine, urine protein, and chromatography of the urinary amino acids in Pascal, Fabrice, Pascal's father, his mother, and his maternal grandparents. All these examinations were normal except a hyperuricemia in the maternal grandfather. This peaked at $115 \mathrm{mg} /$ liter. To determine whether an enzyme deficiency in the purine pathway was at the base of Pascal's difficulty, we examined some of these (see Table 1) in red blood cells.

The activities of guanine and hypoxanthine phosphoribosyl transferase are normal for all the members of the family, whereas the APRTase activity is nil for Pascal and severaly reduced for the mother, the father, and the maternal grandfather.

\section{HYPOTHESIS}

Such a shortage in APRTase (Scheme 2) could impede the reconversion of adenine into adenilic acid and so decrease adenilic acid concentration. As in the hypoxanthine guanine phosphoribosyl transferase (HGPRT) shortage responsible for the LeschNyhan syndrome, this could explain the hyperproduction of uric acid by Pascal, for his APRTase level is almost nil. To our knowledge, a description of a similar case has never been published. Pascal is the product of two apparently healthy parents, both with a reduced level of APRTase and never having gout, uric acid lithiasis, or neurologic troubles. Their respective serum uric acid concentrations are within accepted normal limits.

There is no history of consanguinity between Pascal's mother and father. A partial deficiency in APRTase was found also in Pascal's maternal grandfather, now aged 50 years. He has experienced previous hyperuricemia and urinary lithiasis; the
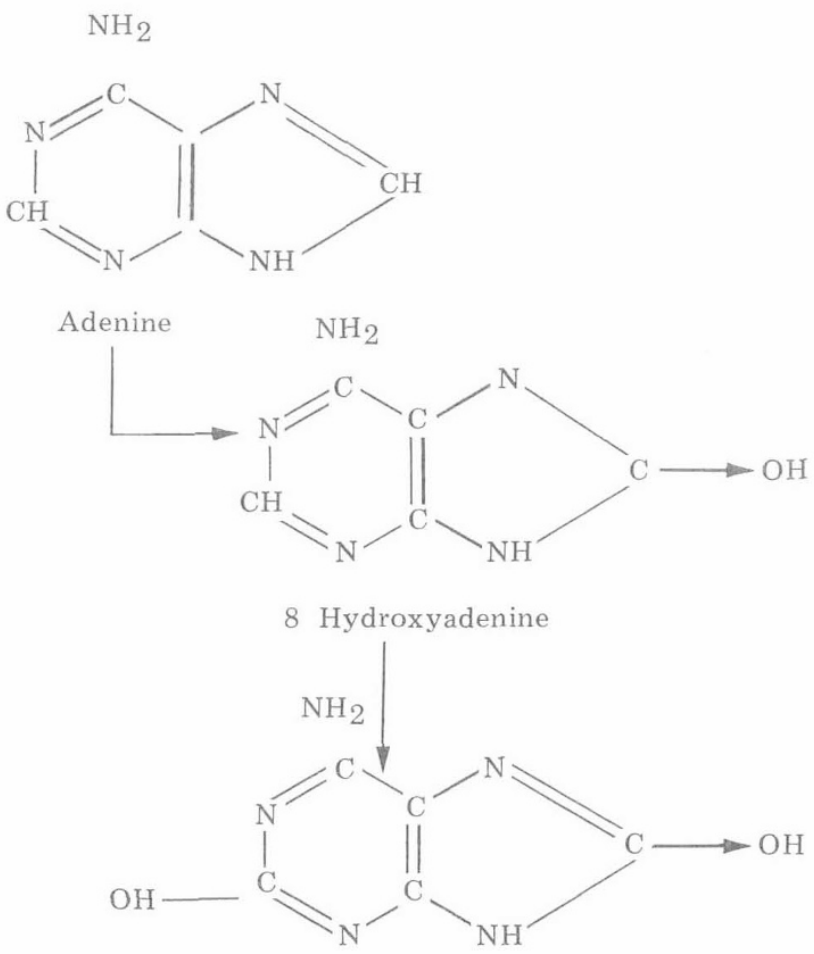

2,8 Dihydroxyadenine

Fig. 1. New catabolism of adenine when deficiency in adenine phosphoribosyl transferase.

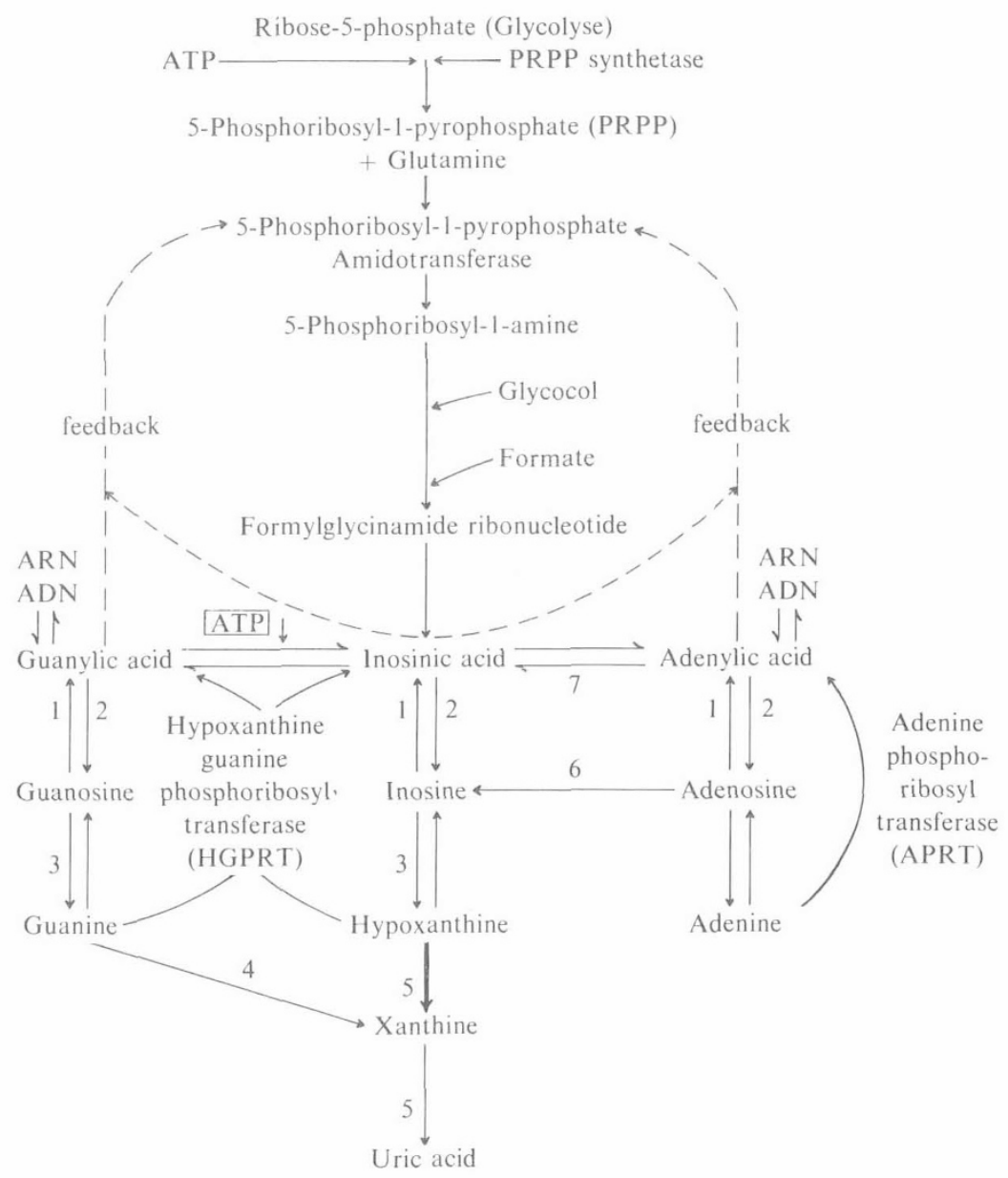

Scheme 2. Purine metabolism pathway. The deficit either in HGPRT or in APRT is accompanied by a chain reaction of the pathway of the de novo synthesis and of a hyperuricemia. From Cartier and Hamet (2). 
00

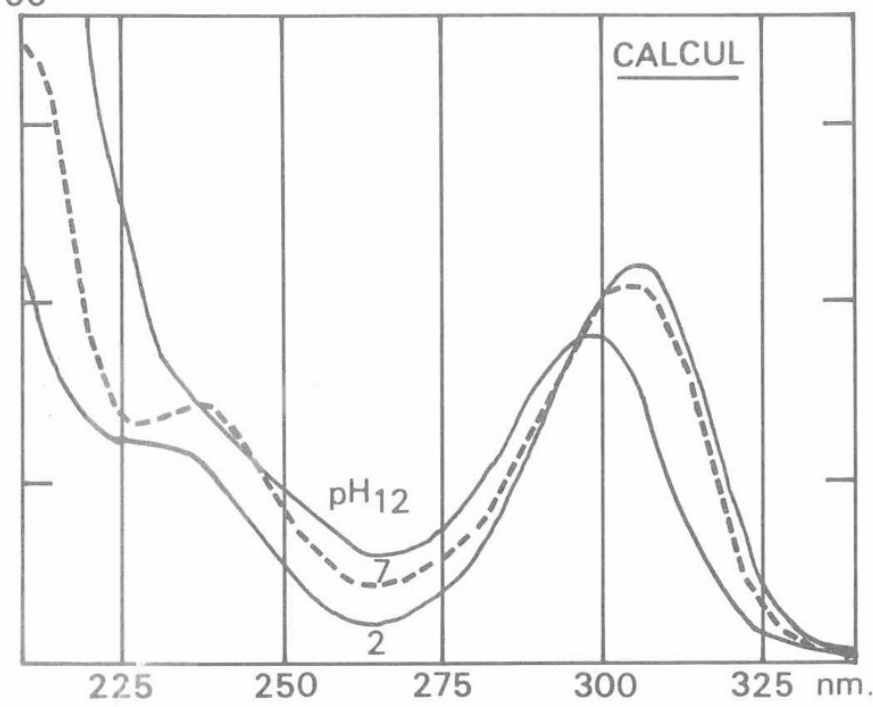

00

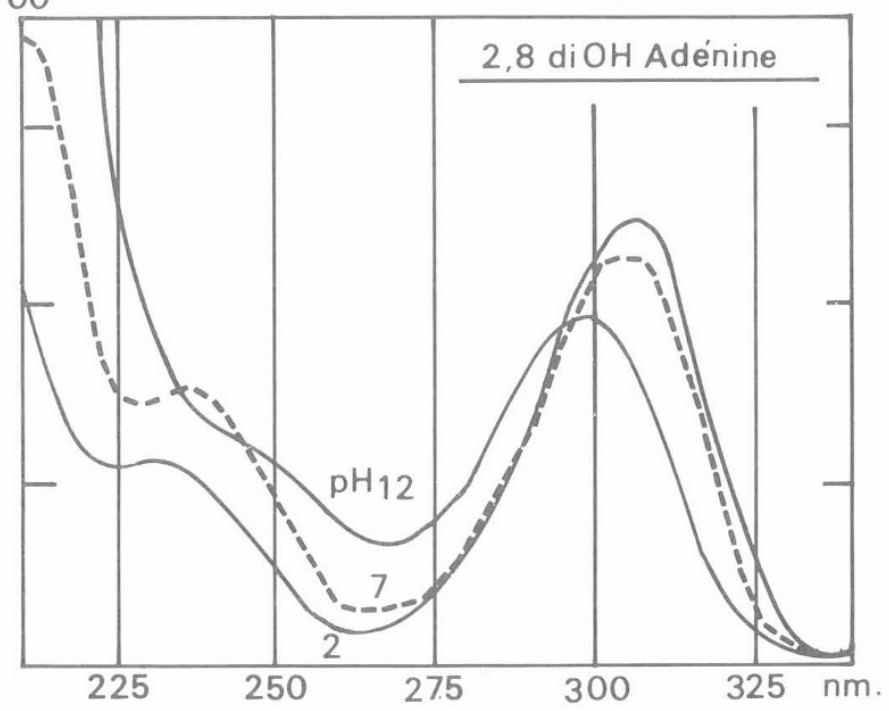

Fig. 2. Ultraviolet spectrum of a calculus (left) and 2,8-dihydroxyadenine (right).

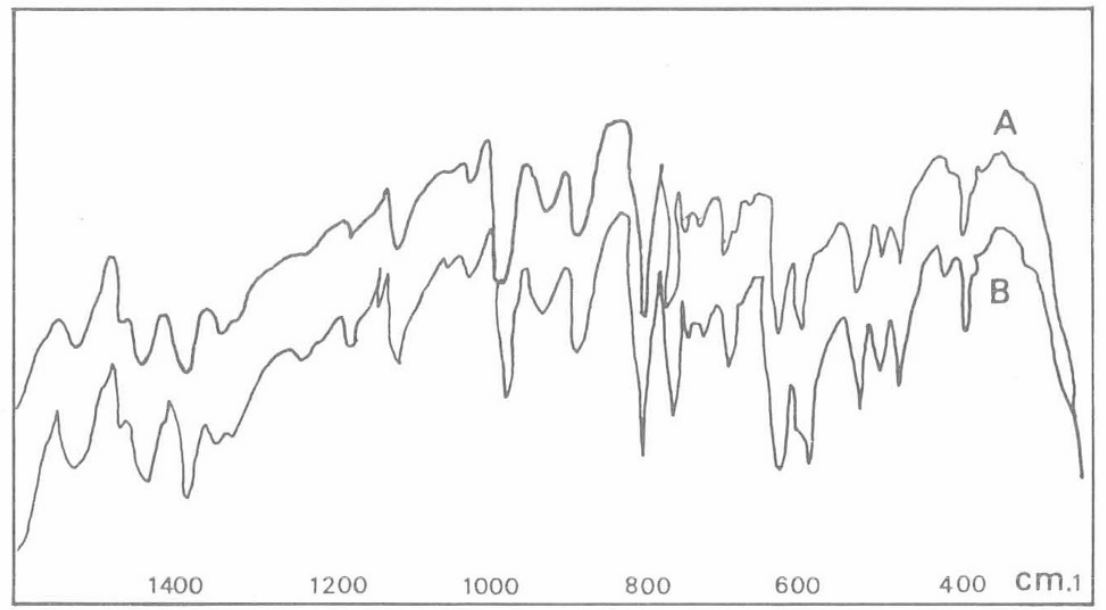

Fig. 3. Infrared spectrum of 2,8-dehydroxyadenine $(A)$ and a calculus $(B)$.

nature of the stones is unknown. When examined by us, his serum uric acid was elevated (115 liter). Thus, it seems likely that Pascal's maternal grandfather and parents represent the heterozygotic form of APRTase deficiency. However, whether a deficiency in this enzyme is responsible for lithiasis cannot be definitively stated at this time. We do know that Pascal's father and mother, who have a relative deficiency, do not demonstrate abnormalities in purine metabolism or form renal calculi.

The characteristics of Pascal's stones and the absence of other associated troubles, particularly neurologic, suggest that a shortage of HGPRT ase is not at fault. Such a shortage is responsible for hyperuricemic encephalopathy of Lesch-Nyhan syndrome hyperuricemia, chronic encephalopathy, a tendency to self-mutilation, and interstitial nephritis of the gout type with or without uric lithiasis (5). The absence of hyperuraturia and true hyperuricemia suggested to us that we should re-examine the calculi. We could not explain the origin of the uric acid lithiasis in the absence of true hyperuricemia or hyperuraturia. Furthermore, the theory that a shortage of APRTase acts by exciting de novo synthesis of purines resulting in stone formation appeared untenable. (It is true that certain partial shortages can also be especially uneventful (4).)

We decided to define the exact nature of the few calculi that we had kept (26 calculi of a total weight of $460 \mathrm{mg}$ ).

The spectrophotometric study of these calculi revealed an absorption spectrum between 210 and $350 \mathrm{~nm}$, suggesting a composition different from uric acid. At $\mathrm{pH} 2$, the maximum absorption at $305 \mathrm{~nm}$ is clearly displaced compared with that of uric acid, at $285 \mathrm{~nm}$. However, the misinterpretation is explainable: on one hand the calculus gives, like uric acid, a positive reaction to murexide and on the other hand it is soluble in alkaline medium. On ultraviolet examination, the composition was found to be $90 \%$ 2,8-dihydroxyadenine; the remaining $5-10 \%$ is uric acid. This identity was strengthned: $(I)$ by comparison with three different samples of 2,8-dihydroxyadenine obtained via synthesis, (2) by oxidation in vitro of the adenine by xanthinoxidase of milk (7), (3) by isolation from kidney of rats poisoned with adenine (1).

The ultraviolet spectrums at various $\mathrm{pH}$ values (Fig. 2), the infrared spectrums (Fig. 3), the spectrums of the x-ray diffraction (Fig. 4) of the calculus and 2,8-dihydroxyadenine are identical. Quantitative analysis was achieved by spectrophotometric measurement after separation on AG $50 \mathrm{~W}-\mathrm{X} 4$ (Fig. 5) or after separation by electrophoresis at $1,500 \mathrm{~V}$ (in $50 \mathrm{mM}$ borate buffer $+1 \mathrm{mM}$ EDT A, pH 9). This compound, scarcely soluble in neutral solution or acid medium ( 50 times less than the uric acid at $\mathrm{pH} 7$ ), precipitates. We have seen in one patient's urine a profuse precipitation of 8 -hydroxyadenine at $2^{\circ}$. The latter certainly constitutes the intermediate between adenine and 2,8-dihydroxyadenine (9). Finally, a part of adenine which is not recuperated is eliminated by the kidney; the urine contains adenine in an appreciable and variable quantity. 

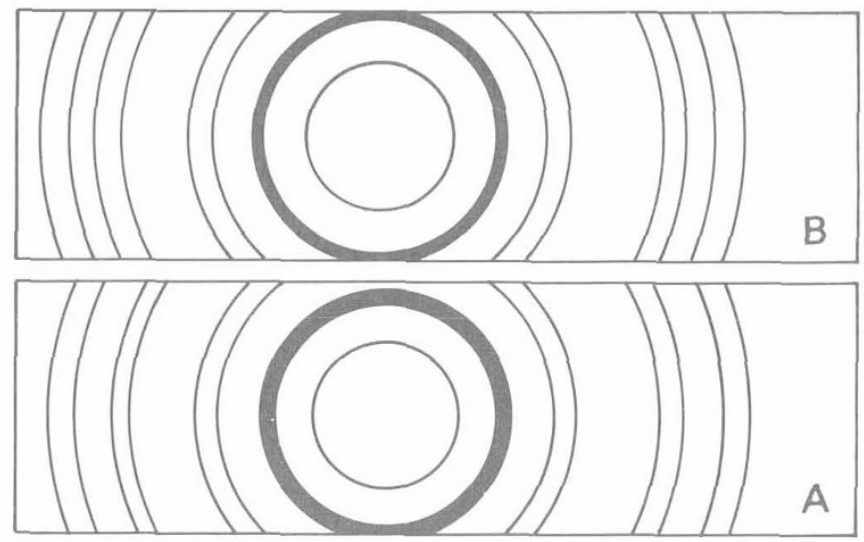

Fig. 4. X-ray diffraction spectrum. $A$ : 2,8-dehydroxyadenine; $B$ : calculus.

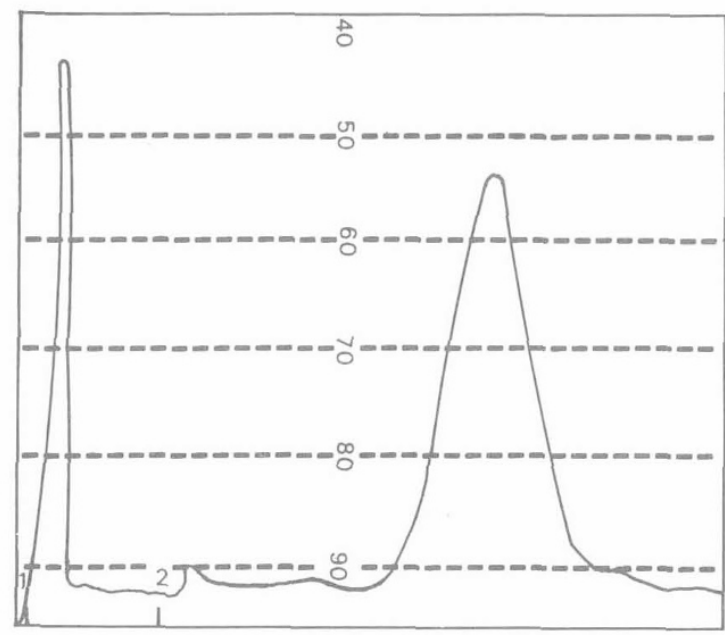

Fig. 5. Chromatographic analysis of a calculus (column, 10 by $150 \mathrm{~mm}$; AG 50-WX4; $200-400$ mesh; elution I water: $2 \mathrm{ClH}$; 0.5 N). Optical density $280 \mathrm{~nm}$. First peak: uric acid; second peak: 2,8-dihydroxyadenine.

\section{SUMMARY}

The spectrophotometric studies allowed us to note that the multiple, precocious (appearing during the first year of life), and recurring urinary lithiasis, associated with a complete deficit of red cell APRTase, was composed of 2,8-dihydroxyadenine and not a uric acid.

Therefore, we feel that this examination and the quantitative analysis of the phosphoribosyl transferases is essential in any case of urinary lithiasis labeled "uric." One must also stress the unique and exceptional nature of this observation, i.e., a complete deficit of APRTase, a homozygotic form resulting from two heterozygotic parents showing no disease. From the therapeutic point of view, certain considerations exist. ( $I$ ) The formation of 2,8-dihydroxyadinine could be slowed by pyrazolopyrimidines such as allopurinol (Ziloric), inhibitors of oxidase xanthine. (2) The solubility of 2,8-dihydroxyadenine differs from that of uric acid. The solubility of the latter quickly increases above $\mathrm{pH}, 7$, whereas that of 2,8-dihydroxyadenine remains very low until pH 9.

Thus alkalinization of urine, used to treat uric acid iithiasis, would be scarcely efficient in a case such as this, if not noxious. This complete deficit in APRTase, by autosomal transmission, appears to lead to an important deviation of the adenine metabolism. Adenine produced during the catabolism of nucleic acids and free nucleotides exists in the intracellular medium only at a very low concentration. Its urinary elimination is almost nil (about 1.5 $\mathrm{mg} /$ day). It is usually directly converted into adenilic acid by APRTase (Km: $\left.0.89-5.210^{-6} \mathrm{M}\right)(8)$.

The absence of adenine phosphoribosyl transferase makes this recovery pathway unoperable.

The adenine is then susceptible to the action of oxidase xanthine which, very likely, oxidizes it in 2,8-dihydroxyadenine at the level of the kidney.

\section{REFERENCES AND NOTES}

1. Bendich, A., Brown, C., Philips, F., and Thiersch, J.: The direct oxidation of adenine in vitro. J. Biol. Chem., 183: 267 (1950).

2. Cartier, P. and Hamet, M. Métabolisme Normal de l'Acide U rique. Actual. Néphrol. Hôpital Necker, 7, 31 (1973).

3. Cavalieri, L., and Bendich, A.: J. Amer. Chem. Soc. 72: 2587 (1950).

4. Delbarre, F., Auscher, C., Amor, B., Degery, A., Cartier, P., and Hamet, M. Gout with adénine phosphoribosyl transferase deficiency. Biomedicine, 21:82 (1974).

5. Forette, B.: Les Enseignements de l'Hyper-uricémie Congénitale. In: Le syndrome de Lesch Nyham. Nouv. Presse Méd., 7: 439 (1973).

6. Kelley, W. N., Fox, I. H., and Wingaarden, J. B.: Further evaluation of adenine phosphoribosyl transferase deficiency in man: Occurrence in a patient with gout. Clin Res., 18: 53 (1970).

7. Klenov, H.: Biochem. J., 50: 404 (1952).

8. Raivio, K., and Seegmiller, J.: In: Current Topics in Cellular Regulation, Vol. 2, p. 201 (Academic Press, New York, 1970).

9. Wingaarden, J., and Dunn, J.: Arch. Biochem. Biophys., 70: 150 (1957).

10. Cartier, P., and Hamet, M.: Une nouvelle maladie métabolique: Déficit complet en adénine phosphoribosyl transferase avec lithiase de 28 di-hydroxyadénine. C. R. Acad. Sci., No. 279 (1973).

11. Requests for reprints should be addressed to: H. DeBray, M.D. Hôpital Saint-Joseph, Service de Pediatrie, 7, Rue Pierre-Larousse, 75674 Paris, Cedex 14 (France).

12. Accepted for publication March 3, 1976. 